\title{
Limited-Sampling Strategies for Anti-Infective Agents: Systematic Review
}

\author{
Denise A Sprague and Mary H H Ensom
}

\begin{abstract}
Background: Area under the concentration-time curve (AUC) is a pharmacokinetic parameter that represents overall exposure to a drug. For selected anti-infective agents, pharmacokinetic-pharmacodynamic parameters, such as AUC/MIC (where MIC is the minimal inhibitory concentration), have been correlated with outcome in a few studies. A limited-sampling strategy may be used to estimate pharmacokinetic parameters such as AUC, without the frequent, costly, and inconvenient blood sampling that would be required to directly calculate the AUC.
\end{abstract}

Objective: To discuss, by means of a systematic review, the strengths, limitations, and clinical implications of published studies involving a limited-sampling strategy for anti-infective agents and to propose improvements in methodology for future studies.

Methods: The PubMed and EMBASE databases were searched using the terms "anti-infective agents", "limited sampling", "optimal sampling", "sparse sampling", "AUC monitoring", "abbreviated AUC", "abbreviated sampling", and "Bayesian". The reference lists of retrieved articles were searched manually. Included studies were classified according to modified criteria from the US Preventive Services Task Force.

Results: Twenty studies met the inclusion criteria. Six of the studies (involving didanosine, zidovudine, nevirapine, ciprofloxacin, efavirenz, and nelfinavir) were classified as providing level I evidence, 4 studies (involving vancomycin, didanosine, lamivudine, and lopinavirritonavir) provided level II-1 evidence, 2 studies (involving saquinavir and ceftazidime) provided level II-2 evidence, and 8 studies (involving ciprofloxacin, nelfinavir, vancomycin, ceftazidime, ganciclovir, pyrazinamide, meropenem, and alpha interferon) provided level III evidence. All of the studies providing level I evidence used prospectively collected data and proper validation procedures with separate, randomly selected index and validation groups. However, most of the included studies did not provide an adequate description of the methods or the characteristics of included patients, which limited their generalizability.

Conclusions: Many limited-sampling strategies have been developed for anti-infective agents that do not have a clearly established link between AUC and clinical outcomes in humans. Future studies should first determine if there is an association between AUC monitoring and clinical outcomes. Thereafter, it may be worthwhile to prospectively develop and validate a limited-sampling strategy for the particular anti-infective agent in a similar population.

Key words: limited-sampling strategy, anti-infectives, pharmacokinetics, therapeutic drug monitoring

\section{RÉSUMÉ}

Contexte : Laire sous la courbe de la concentration en fonction du temps (ASC) est un paramètre pharmacocinétique qui représente l'exposition globale d'un patient à un médicament. En ce qui a trait à des agents anti-infectieux sélectionnés, les paramètres pharmacocinétiques et pharmacodynamiques, comme l'ASC/CMI (concentration minimale inhibitrice), ont été corrélés avec les résultats cliniques dans un nombre limité d'études. On peut utiliser une stratégie de prélèvements limités pour estimer la valeur des paramètres pharmacocinétiques, comme l'ASC, sans avoir à recourir aux prélèvements de sang fréquents, coûteux et peu pratiques qui sont nécessaires pour calculer directement l'ASC.

Objectif : Discuter, au moyen d'une analyse systématique, les forces, les limites et les implications cliniques des études publiées comportant une stratégie de prélèvements limités pour les agents anti-infectieux et proposer des améliorations à la méthodologie de futures études.

Méthodes : Les bases de données PubMed et EMBASE ont été interrogées en utilisant les termes " agents anti-infectieux » (" anti-infective agents »), " prélèvements limités " (" limited sampling »), "prélèvements optimaux " (" optimal sampling »), " prélèvements parcimonieux " (" sparse sampling »), surveillance de l'ASC ("AUC monitoring "), ASC abrégée (" abbreviated AUC »), prélèvements simplifiés (" abbreviated sampling ») et bayésien ("Bayesian »). Les listes de référence des articles extraits ont été examinées manuellement. Les études retenues ont été classées selon des critères modifiés du US Preventive Services Task Force.

Résultats : Vingt études ont satisfait aux critères d'inclusion. Six de ces études (portant sur la didanosine, la zidovudine, la névirapine, la ciprofloxacine, l'éfavirenz et le nelfinavir) ont été classées comme fournissant des données probantes de niveau I, quatre études (portant sur la vancomycine, la didanosine, la lamivudine et le lopinavir-ritonavir) ont fourni des données probantes de niveau II-1, deux études (portant sur le saquinavir et la ceftazidime) ont fourni des données probantes de niveau II-2, et huit études (portant sur la ciprofloxacine, le nelfinavir, la vancomycine, la ceftazidime, le ganciclovir, la pyrazinamide, le méropenem et l'interféron alpha) ont fourni des données probantes de niveau III. Toutes les études fournissant des données probantes de niveau I ont utilisé des données recueillies prospectivement et des méthodes de validation adéquates avec un groupe de référence et un groupe de validation distincts et choisis au hasard. Cependant, la plupart des études incluses n'ont pas fourni une description adéquate des méthodes ou des caractéristiques des patients admis, ce qui a limité leur généralisabilité.

Conclusions : Plusieurs stratégies de prélèvements limités ont été développées pour les agents anti-infectieux et elles ne comportent pas de lien clairement établi entre l'ASC et les résultats cliniques chez l'humain. Les études ultérieures devraient d'abord déterminer s'il existe un lien entre la surveillance de l'ASC et les résultats cliniques. Il pourra par la suite 

prélèvements limités pour un agent anti-infectieux particulier chez une population de patients similaire.

Mots clés : stratégies de prélèvements limités, agents anti-infectieux, pharmacocinétique, surveillance thérapeutique pharmacologique

[Traduction par l'éditeur]

\section{INTRODUCTION}

$\mathrm{I}$ has been proposed that therapeutic drug monitoring is warranted when a drug exhibits a narrow therapeutic range, therapy is of sufficient duration, pharmacokinetic parameters have been correlated with clinical outcome, the pharmacodynamic response is not readily assessable, and/or the drug assay results provide more information than clinical judgement alone. ${ }^{1}$ For a drug that is suitable for therapeutic drug monitoring, measurement of the area under the concentrationtime curve (AUC) is considered a good representation of overall exposure to the drug. For selected anti-infective agents, pharmacokinetic parameters such as AUC/MIC (drug exposure relative to the bacterial minimum inhibitory concentration) and peak/MIC (peak concentration relative to the bacterial minimum inhibitory concentration) have been correlated with outcomes in animal, in vitro, and a small number of human studies. ${ }^{2}$ In their review of pharmacokinetic and pharmacodynamic considerations for selecting agents for outpatient parenteral antimicrobial therapy, Slavik and Jewesson $^{2}$ discussed several anti-infectives for which AUC/MIC may correlate with clinical efficacy. Such agents include, but are not limited to, fluoroquinolones (ciprofloxacin, levofloxacin, gatifloxacin) and quinupristin-dalfopristin. ${ }^{2}$

The use of AUC is limited, however, by the large number of blood samples required for its accurate determination. As many as 10 or more samples may be needed to characterize AUC in the research setting, but in clinical practice such frequent blood sampling is impractical, time-consuming, costly, and, for infants, potentially unethical. It also may not be possible to obtain blood samples frequently from elderly or critically ill patients with poor venous access.

One proposed method of reducing the cost and inconvenience of frequent sampling is the use of limited-sampling strategies. A limited-sampling strategy is a method of characterizing pharmacokinetic parameters, particularly the AUC, using relatively few blood samples, usually 3 or fewer. The methods used to develop and validate limited-sampling strategies have been reviewed elsewhere. ${ }^{3-7}$ Briefly, these approaches are usually developed using either multiple regression analysis with a stepwise approach or population estimates with the Bayesian approach. Multiple regression analysis determines the relation between the dependent variable (usually AUC) and various independent variables (i.g., timed concentrations from serially collected blood samples). The resulting limited-sampling strategy is described as follows:
$\mathrm{AUC}=b+m_{1} C\left(t_{1}\right)+m_{2} C\left(t_{2}\right)+m_{3} C\left(t_{3}\right)+\ldots m_{i} C\left(t_{i}\right)$ where $C(t)$ is the drug concentration at time $t_{i}, b$ represents the $y$-intercept, and $m_{i}$ represents the slope of the equation at time $t_{i}{ }^{3-7}$ The Bayesian method blends population estimates with data from individual patients; as such, both population and individual data are required. If population estimates are unavailable, the index data set can be used to determine them. Then, one or more timed concentrations from the validation data set are entered as individual data to predict the AUC. Equations with a high coefficient of determination $\left(r^{2}\right)$ are typical candidates for a limited-sampling strategy, which is then subjected to testing with a validation data set. ${ }^{3-7}$ Acceptable methods for validation include data splitting (ideally by randomly assigning patients to index and validation groups), cross-validation (multiple data splitting), jackknife resampling, and bootstrap resampling. Bias and the precision of limitedsampling strategies are often presented as mean prediction error and mean squared prediction error, according to the methods of Scheiner and Beal. ${ }^{8}$ A commonly accepted range of bias and precision values is $15 \%$ to $20 \%$. Another important requirement of a clinically useful limited-sampling strategy would be a maximum of 3 conveniently timed (e.g., obtained within $4 \mathrm{~h}$ after administration of a dose) concentrations. ${ }^{3-7}$

The objectives of this review were to critically evaluate published limited-sampling strategies for anti-infective agents, to discuss the clinical implications of these strategies as they apply to anti-infectives, and to propose improvements in methodology for future studies of limited-sampling strategies.

\section{METHODS}

The PubMed (January 1966 to December 2008) and EMBASE (January 1980 to December 2008) databases were searched to identify potential studies for review. The following search terms were used: "anti-infective agents", "limited sampling", "optimal sampling”, "sparse sampling”, "AUC monitoring", "abbreviated AUC", "abbreviated sampling”, and "Bayesian". The reference lists of retrieved articles were also searched manually.

Studies were retrieved if the abstract described the use of limited, optimal, or sparse blood sampling for monitoring an anti-infective agent. Studies published in abstract form were excluded. Studies published in full were included if they described the development of limited-sampling strategies to predict AUC or peak concentrations for anti-infective agents in humans and were written in English. Studies conducted in 
healthy volunteers or in patients without an active infection were excluded; the results of such studies cannot be extrapolated to patients with active infections because of potential differences in pharmacokinetic-pharmacodynamic parameters. Also excluded were studies that did not suggest sampling times and those that merely described a previously developed and validated limited-sampling strategy.

Included studies were classified according to their levels of evidence. Because there are no formalized criteria for determining levels of evidence for studies of limited-sampling strategies, the criteria were adapted from those developed by the US Preventive Services Task Force.' This adaptation has been used successfully in previous reviews of limited-sampling strategies of immunosuppressants ${ }^{5}$ and chemotherapy agents. ${ }^{6,7}$ Studies were classified into 4 categories (level I, II-1, II-2, or III) according to criteria presented in Table $1 .^{5-7,9}$

\section{RESULTS}

Thirty-four studies met the initial inclusion criteria. ${ }^{10-44}$ Fourteen of these studies ${ }^{10-24}$ were excluded: 4 were conducted in healthy volunteers, ${ }^{10-13} 1$ involved volunteers with cystic fibrosis who did not have an active infection, ${ }^{14} 8$ did not suggest sampling times, ${ }^{14-22}$ and 2 studied previously validated limited-sampling strategies in new populations. ${ }^{23,24}$ Table 2 summarizes the characteristics of the included studies ${ }^{25-44}$ according to their levels of evidence. The following information was extracted from each study: level of evidence, the antiinfective agent, the population for derivation of the limitedsampling strategy, the sampling times investigated, the suggested timed samples, the equation(s) for the limited-sampling strategy, $r$ (the correlation coefficient) or $r^{2}$, the percent bias for the validation group, the percent precision, and additional comments.
Two studies for each of the following drugs described limited-sampling strategies (Table 2): ciprofloxacin (level I and level III evidence, respectively), didanosine (level I and level II1 evidence, respectively), nelfinavir (level I and level III evidence, respectively), vancomycin (level II-1 and level III evidence, respectively) and ceftazidime (level II-2 and level III evidence, respectively). Limited-sampling strategies were described in a single study for each of the following agents: zidovudine (level I evidence), nevirapine (level I evidence), efavirenz (level I evidence), lamivudine (level II-1 evidence), lopinavir-ritonavir (level II-1), saquinavir (level II-2 evidence), ganciclovir (level III evidence), pyrazinamide (level III evidence), meropenem (level III evidence) and alpha interferon (level III evidence).

\section{DISCUSSION}

\section{Study Strengths and Limitations}

Six studies of limited-sampling strategies were considered to present evidence of the highest quality (level I), describing strategies for didanosine, ${ }^{25}$ zidovudine, ${ }^{26}$ nevirapine, ${ }^{27}$ ciprofloxacin, ${ }^{28}$ efavirenz, ${ }^{29}$ and nelfinavir ${ }^{30}$ (Table 2). Each study used prospectively collected data and proper validation procedures. All 6 studies randomized pharmacokinetic data into index and validation groups, and 5 of the studies clearly randomized the pharmacokinetic data into independent data sets. ${ }^{25,27-30}$ Each study illustrated the potential utility of limitedsampling strategies by requiring only 1 or 2 blood samples to predict AUC, with minimal bias and relatively good precision. The level I studies of didanosine and zidovudine also provided 1-sample limited-sampling strategies to predict a second pharmacokinetic parameter, maximum drug concentration $\left(C_{\max }\right) \cdot 25,26$

Of the level I studies, the study of a validated limitedsampling strategy for nevirapine probably provided the most

\section{Table 1. Classification of studies of limited sampling strategies ${ }^{5-7,9}$}

\section{Level of Evidence}

Level I (evidence obtained from at least one properly

randomized controlled trial)

\section{Description}

- Used prospectively collected data

- Had proper validation procedures (separate validation group, jackknife method, or bootstrap method)

- Assigned data randomly into index and validation groups (not applicable when using jackknife and bootstrap methods)

- Studies assumed to be prospective even if not explicitly stated by authors

Level II-1 (evidence obtained from well-designed controlled trials without randomization)

Level II-2 (evidence obtained from well-designed cohort or case-control analytic studies, preferably from more than one centre or research group)

Level III (opinions of respected authorities,

based on clinical experience; descriptive studies and

case reports; or reports of expert committees)
- Used prospectively collected data

- Had proper validation procedures

- Lacked random assignment of data into index and validation groups

- Used retrospective data

- Had proper validation procedures regardless of randomization

- Did not have proper validation procedures 


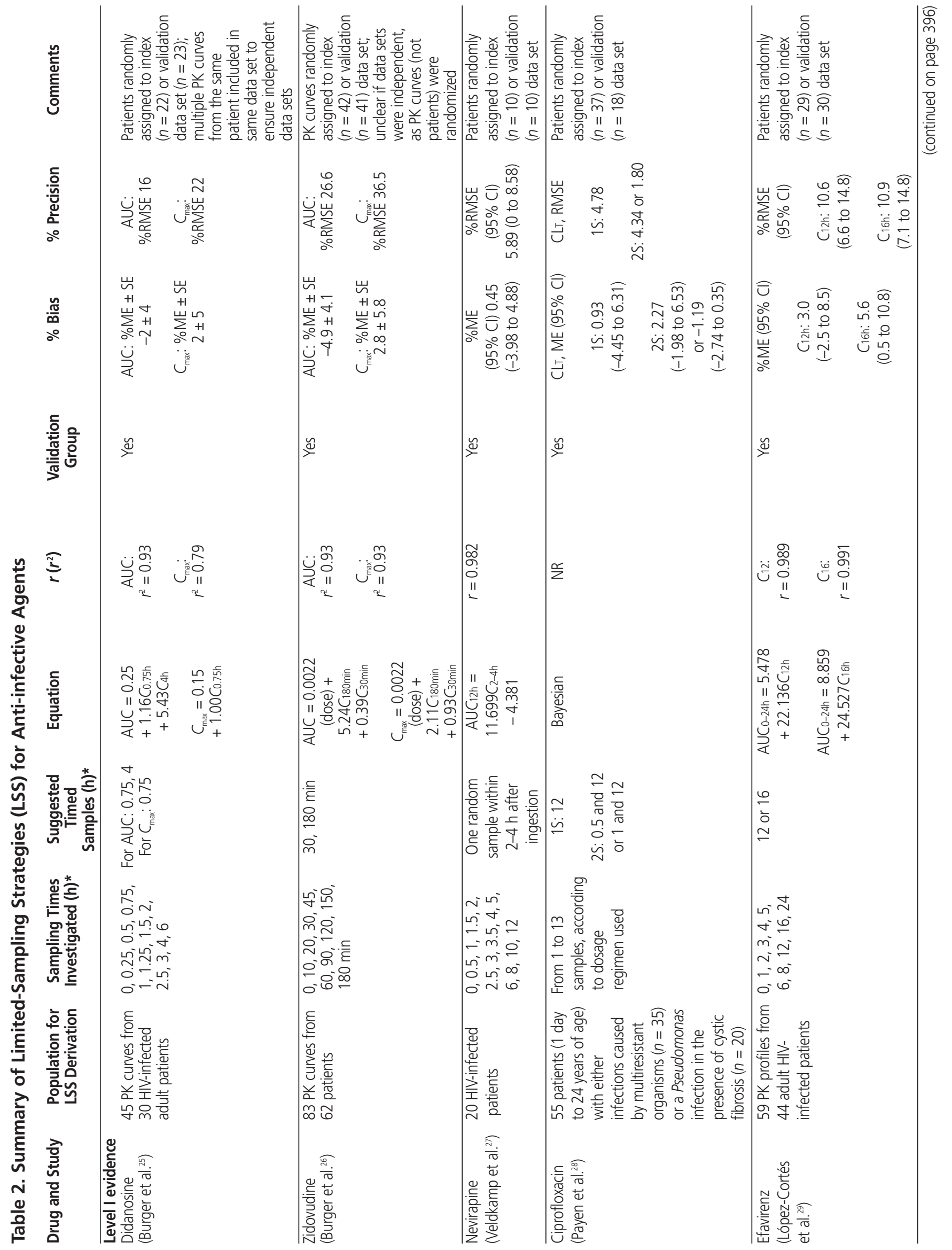




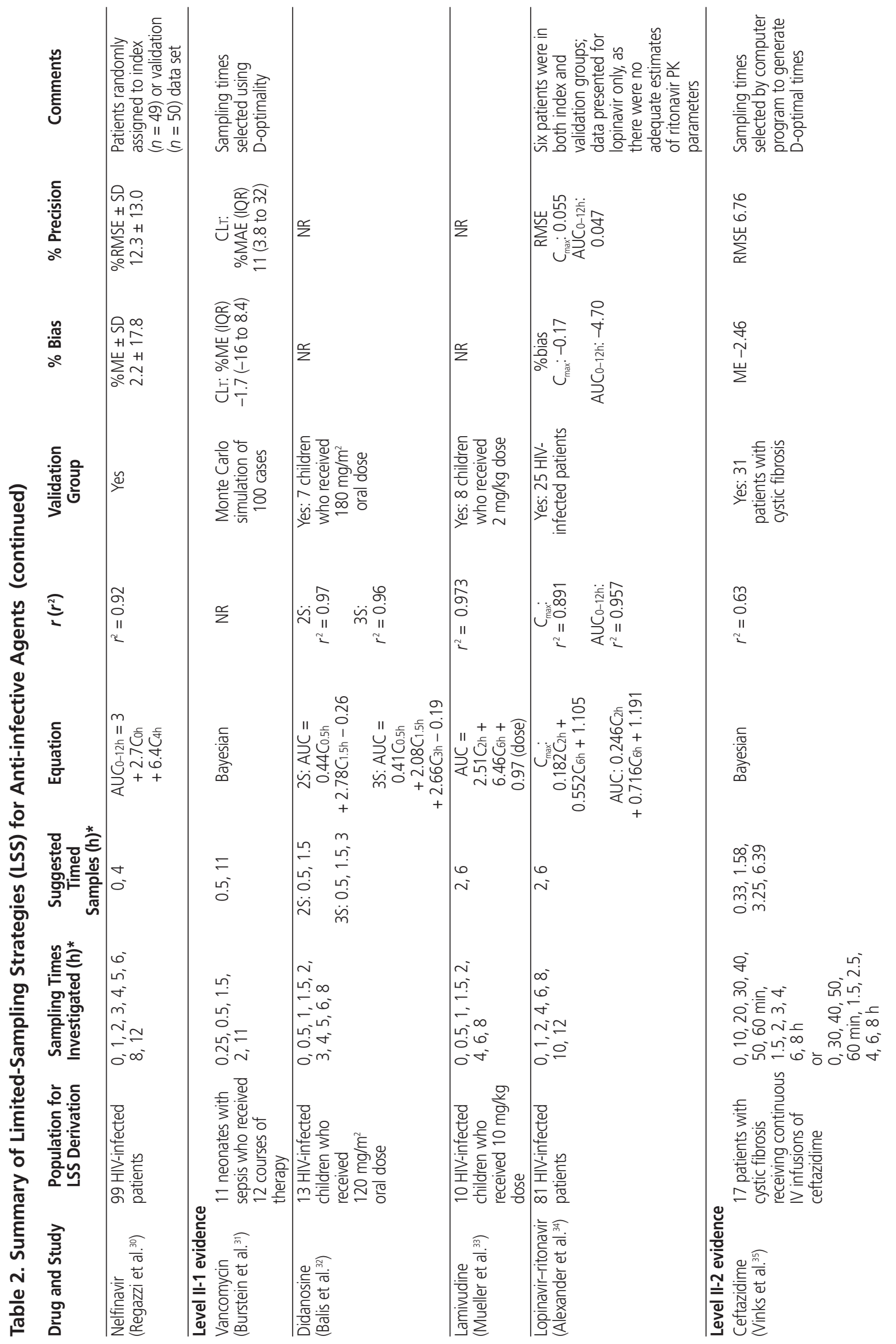




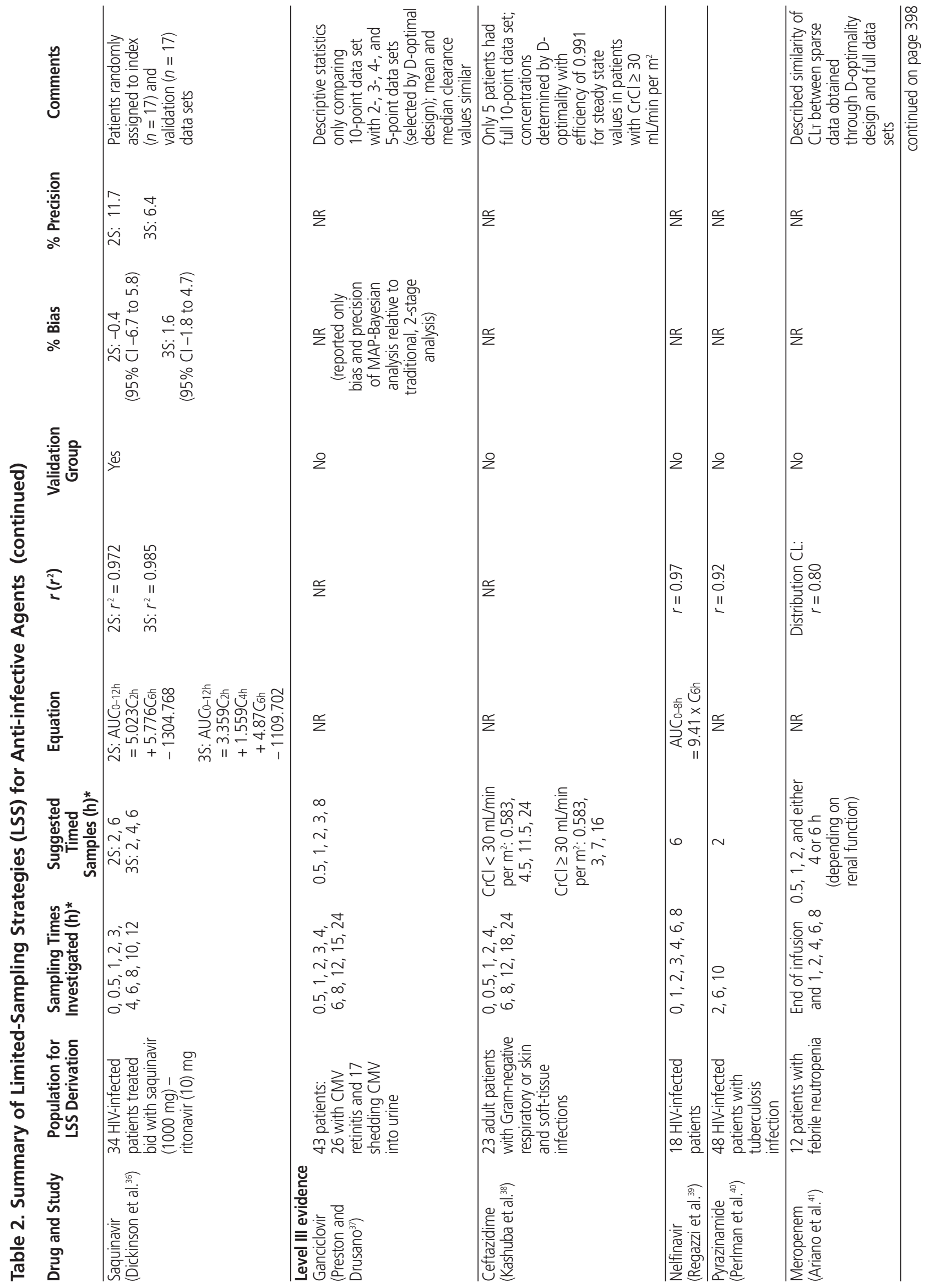




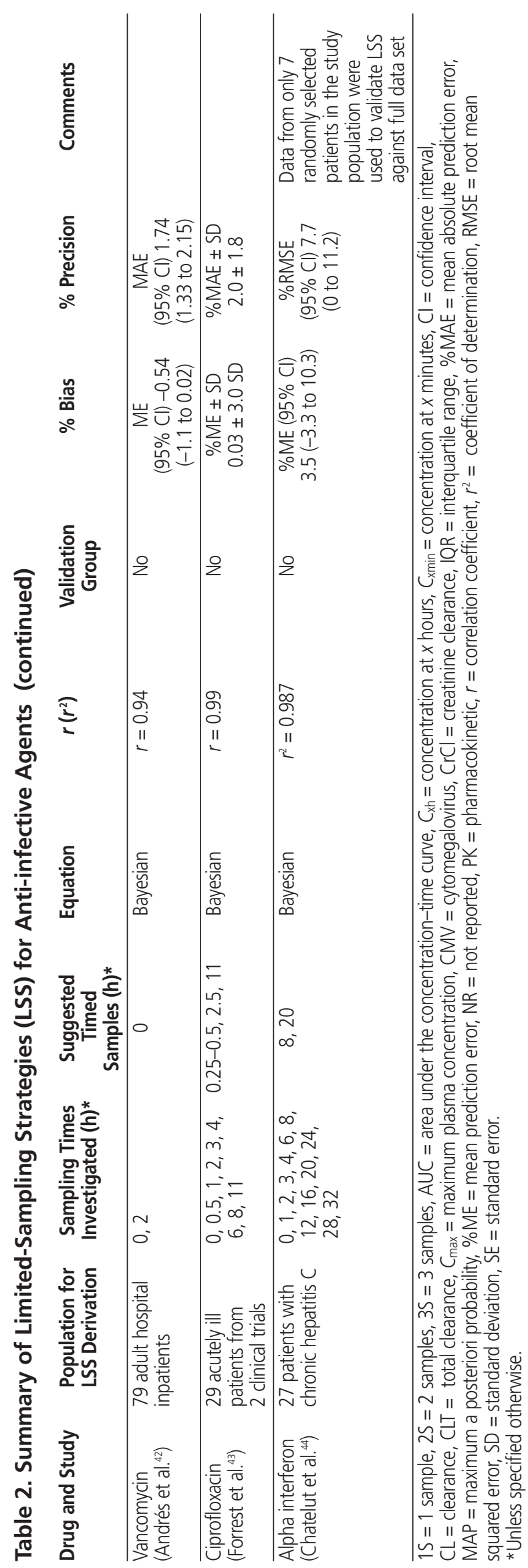

flexibility and convenience for clinical use. ${ }^{27}$ In that study, all 14 sampling data points were used to determine a 1 -sample (i.e., a random sample between 2 and $4 \mathrm{~h}$ after dosing) limitedsampling strategy that predicted the AUC with minimal bias and good precision. ${ }^{27}$ This single "random" sample would be a convenient method for future research to determine if the AUC for nevirapine correlates with clinical outcome. A distinctive strength of the efavirenz study was that concomitant medications were accounted for in the randomization scheme. ${ }^{29}$ Of the level I studies, the nelfinavir trial had the largest sample size, with random assignment of $99 \mathrm{HIV}$-infected patients to the index $(n=49)$ and validation $(n=50)$ groups. $^{30}$

Five anti-infective agents were each studied in 2 separate trials, as described in Table 2. $25,28,30-32,3,3,38,39,42,43$ Classification according to level of evidence is important when there are discrepant results between studies for suggested sampling times to characterize pharmacokinetic parameters. As a general guideline, clinicians may choose to place more weight on results of studies classified as having a higher level of evidence. For example, although the 2 ceftazidime studies suggested 2 different 4-point sampling strategies to characterize AUC, one study had level II-2 evidence ${ }^{35}$ and the other had level III evidence. $^{38}$ The 2 didanosine studies also yielded discordant limited-sampling strategies. ${ }^{25,32}$ Although this may have been due to differences in the populations studied (adults versus children), one study had level I evidence and the other level II-1 evidence. The 2 studies that described limited-sampling strategies for ciprofloxacin also produced discordant results. ${ }^{28,43}$ The level III study required an additional sample at $2.5 \mathrm{~h}$ to best characterize total clearance. ${ }^{43}$ However, when restricted to only 2 samples, the selected times were similar to those suggested in the level I study. ${ }^{28}$ The 2 nelfinavir studies were difficult to compare, as they characterized 2 different pharmacokinetic parameters $\left(\mathrm{AUC}_{0-12 \mathrm{~h}}\right.$ and $\left.\mathrm{AUC}_{0-8 \mathrm{~h}}\right)$ and provided level I and level III evidence, respectively. ${ }^{30,39}$ The 2 vancomycin studies also developed limited-sampling strategies for prediction of different parameters (clearance and concentration), but again were classified as providing level II-1 and level III evidence, respectively. ${ }^{31,42}$

In general, the studies identified in this systematic review had small sample sizes, the methods and patient populations were not well described, and a variety of methods were used to determine optimal sampling times (Table 2). Two studies 37,41 did not report bias or precision for the variations in sampling times used in development of the limited-sampling strategy; instead, only descriptive statistics of clearance were provided. Methods for determining the sampling times to be used in the limited-sampling strategies included arbitrarily selecting times ${ }^{43}$ and use of software to obtain D-optimal times. ${ }^{31,35,37,38}$ Use of a computer software program to determine optimal sampling times may yield times that are impractical, as was the case for one ceftazidime study. ${ }^{35}$

One study, considered to report level I evidence, randomized data from 83 pharmacokinetic curves into separate index 
and validation groups, but it is unclear if the 2 groups represented 2 independent sets of patients, as the data were obtained from only 62 patients. ${ }^{26}$ The study also did not provide information about the baseline characteristics of the patients, so it was assumed that the "patients" who "ingested their usual morning dose" were receiving zidovudine therapy for HIV and were not volunteers. ${ }^{26}$ It would be difficult to apply this limited-sampling strategy in practice, as little is known about the underlying population that was studied. It has been previously shown that a limited-sampling strategy for a drug cannot always be extrapolated to a population other than the one studied. For example, a limited-sampling strategy for didanosine was evaluated in $13 \mathrm{HIV}$-infected children in a study with level II-1 evidence, ${ }^{32}$ but the recommended equations did not reliably predict the AUC in a study of HIVinfected adults. ${ }^{25}$ In addition to the expected differences in pharmacokinetics between adults and children, this discrepancy might also have resulted from a difference in the rigour of the evaluations.

Three studies, all considered to present level I evidence, did not report concomitant medications or medical conditions, which limits the generalizability of their findings. ${ }^{25,27,28}$ In studies of antiretroviral agents, which have numerous potential pharmacokinetic interactions, it would be important to note concomitant antiretrovirals and other medications. In the studies with level I evidence that were identified in this review, patients were prospectively and randomly assigned to index and validation groups, but because the studies were small, it is possible that not all characteristics were balanced between the groups.

A ciprofloxacin study of level I evidence $(n=55)$ included 20 patients with cystic fibrosis, all less than 25 years of age. ${ }^{28}$ Given the pharmacokinetic characteristics specific to patients with cystic fibrosis, such as increased clearance, as well as the pharmacokinetic differences across pediatric and adolescent age groups, it would be desirable to develop and validate a limitedsampling strategy in a study of patients with cystic fibrosis within a narrower age range. The ciprofloxacin stud $y^{28}$ also used 4 different sets of sampling times, ranging from 1 to 13 samples, and selected times on the basis of the dosing regimen used. It would be preferable to characterize a full pharmacokinetic profile for several patients with similar characteristics, all of whom received the same dose, and to attempt validation for all possible combinations of sampling times, to obtain the most precise and least biased limited-sampling strategy. A level I study of a limited-sampling strategy for efavirenz exemplified proper validation procedures, in that patients were randomly assigned to 3 different sets of index and validation groups according to concomitant interacting medications. ${ }^{29}$ However, that study also assessed only 3 single sampling times in its evaluation. Although full pharmacokinetic profiles were obtained, the reason for the authors' choice of 3 time points $(8,12$, and $16 \mathrm{~h})$ to estimate the AUC and trough (at $24 \mathrm{~h})$ was not stated. ${ }^{29}$
A study of nelfinavir-treated patients, which had level I evidence, provided some baseline comparative information on comorbid conditions between index and validation groups; it also had a more complete evaluation of sampling times than the other level I studies. ${ }^{30}$ However, little information on concomitant medication was provided, and, as discussed below, the use of nelfinavir has now fallen out of favour.

\section{Clinical Implications}

It is difficult to draw conclusions from the limitedsampling strategies that have been described in the literature to date, given their methodologic flaws and limitations. As well, discrepancies in results between studies may be attributable to the differences between the patient subpopulations being studied. For example, in addition to pathophysiologic parameters (e.g., age, sex, disease states), results for limitedsampling strategies may vary according to dosing schedules, drug bioavailability, and other pharmacokinetic parameters such as elimination half-life. More importantly, there is a lack of evidence supporting the need for therapeutic drug monitoring for the majority of anti-infectives for which limited-sampling strategies have been developed. In other words, even if clinical efficacy and AUC are related, a limited-sampling strategy may be of limited clinical utility. For example, concentrations of the non-nucleoside reverse transcriptase inhibitors (NNRTIs) nevirapine and efavirenz are not routinely monitored in practice, because clinicians are able to monitor efficacy and toxicity clinically and the evidence related to therapeutic drug monitoring for these agents is conflicting. ${ }^{45}$ Therapeutic drug monitoring of the nucleoside reverse transcriptase inhibitors, such as didanosine, stavudine, zidovudine, and lamivudine, is also not routine practice. ${ }^{46,47}$ These agents require intracellular activation, and the intracellular concentration of active drug does not correlate well with the plasma concentration of the parent compound.

Although correlation between pharmacokineticpharmacodynamic data and microbiological cure has been demonstrated in vitro and in animal models, there are limited prospective human data correlating pharmacokinetic-pharmacodynamic parameters with clinical outcomes. For the ß-lactam anti-infectives, such as ceftazidime and meropenem, which were included in this review, it appears that time above MIC $(t>$ MIC) is actually the pharmacokinetic-pharmacodynamic parameter that correlates best with microbiological and clinical efficacy. ${ }^{2}$ The $t>$ MIC parameter represents the time that the antibiotic concentration remains above a certain threshold concentration, usually a concentration 4 to 5 times greater than the MIC. These data are again based largely on animal and in vitro data. However,, if $t>\mathrm{MIC}$ is the parameter that correlates best with efficacy, as has been traditionally thought for the timedependent $B$-lactams, determining the AUC would not be required. Therefore, limited-sampling strategies for the $ß$-lactam anti-infectives would not be necessary. 
Two studies included in this review describe limitedsampling strategies for vancomycin. Although vancomycin peak and trough concentrations have been routinely monitored for years, there are limited human data to support an association between concentration and efficacy or toxicity. ${ }^{48}$ The AUC/MIC has been correlated with improved outcome in animal models and one human study, but other unpublished human data indicate no relationship between the pharmacokinetic-pharmacodynamic parameters and efficacy.

Plasma concentrations of protease inhibitors may be associated with efficacy and toxicity. However, 2 of the protease inhibitors included in studies of limited-sampling strategies may now be less frequently used in practice. It is now known that ethyl methanesulfonate, an animal carcinogen and teratogen, is released in small amounts during the manufacturing of nelfinavir. ${ }^{47}$ This has led to an advisory against using nelfinavir in pregnancy and withdrawal of the drug from the market in some countries. Saquinavir may also be used less frequently than other protease inhibitors because of a greater "pill burden" and the requirement for twice-daily dosing. ${ }^{47}$ In addition, the efficacy and toxicity of these agents can be monitored clinically, on the basis of viral load, CD4 count, physical symptoms, and laboratory parameters, as is the case of the NNRTIs. This may limit the utility of therapeutic drug monitoring for these agents.

Data also suggest that AUC/MIC and peak/MIC may be correlated with the efficacy of the fluoroquinolones. ${ }^{2}$ These data have been derived mostly from animal and in vitro modelling, but a few retrospective and observational human studies support these findings as well. However, therapeutic drug monitoring is not routine practice for the fluoroquinolones, because the efficacy and toxicity of these drugs can be monitored clinically.

\section{Suggestions for Future Studies}

To date, AUC/MIC has been suggested to correlate with clinical efficacy for a limited number of anti-infective agents (e.g., fluoroquinolones, quinupristin-dalfopristin). ${ }^{2}$ Ideally, limitedsampling strategies would be developed only for those drugs for which the need for therapeutic drug monitoring has been clearly demonstrated. Alternatively, any limited-sampling strategies that are developed should be assessed to determine if their use results in better clinical outcomes than usual practice (i.e., no monitoring). Optimally, limited-sampling strategies should be prospectively evaluated in a large number of patients randomly assigned to either an index group or a validation group, to determine the validity of the model. Blood sampling in the index group would need to be sufficient to adequately characterize the AUC. Then, all possible combinations of limited sampling at convenient times, using 3 or fewer samples, would be tested to determine the optimal sampling times to characterize AUC or other pharmacokinetic parameters. In addition, the characteristics of the included patients should be clearly outlined to establish generalizability. If it is desirable to use the limited-sampling strategy in a population other than the one studied, the method must first be validated in the new population.

\section{Conclusions}

To our knowledge, this is the first systematic review of limited-sampling strategies for anti-infective agents. The findings indicate that although a number of such strategies have been developed, the important link between limited-sampling strategies and clinical outcomes has not yet been established. Despite the identification of 6 level I studies in this review, it is difficult to draw conclusions from the majority of studies of limited-sampling strategies that have been reported in the literature to date, given their methodologic flaws and the limited data correlating pharmacokinetic-pharmacodynamic monitoring with clinical outcomes of anti-infective therapy. Future studies should first determine if monitoring pharmacokineticpharmacodynamic parameters yields better predictions of efficacy and/or toxicity of an anti-infective agent than no monitoring at all. Once an association between AUC monitoring and clinical outcomes has been clearly established, it may be worthwhile to prospectively develop and evaluate a limitedsampling strategy for the particular anti-infective agent in a similar population.

\section{References}

1. Ensom MHH, Davis GA, Cropp CD, Ensom RJ. Clinical pharmacokinetics in the 21st century. Does the evidence support definitive outcomes? Clin Pharmacokinet 1998;34(4):265-279.

2. Slavik RS, Jewesson PJ. Selecting antibacterials for outpatient parenteral antimicrobial therapy: pharmacokinetic-pharmacodynamic considerations. Clin Pharmacokinet 2003;42(9):793-817.

3. Ette EI, Williams PJ, Lane JR. Population pharmacokinetics III: design, analysis and application of population pharmacokinetic studies. Ann Pharmacother 2004;38(12):2136-2144.

4. Brendel K, Dartois C, Comets E, Lemenuel-Diot A, Laveille C, Tranchand $\mathrm{B}$, et al. Are population pharmacokinetic and/or pharmacodynamic models adequately evaluated? A survey of the literature from 2002 to 2004. Clin Pharmacokinet 2007;46(3):221-234.

5. Ting LSL, Villeneuve E, Ensom MHH. Beyond cyclosporine: a systematic review of limited sampling strategies for other immunosuppressants. Ther Drug Monit 2006;28(3):419-430.

6. Loh GW, Ting LSL, Ensom MHH. A systematic review of limited sampling strategies for platinum agents used in cancer chemotherapy. Clin Pharmacokinet 2007;46(6):471-494.

7. Pattar R, Ensom MHH. Utility of limited sampling strategies for anticancer agents in the clinical arena: a systematic review. Curr Cancer Ther Rev 2009;5(1):45-66.

8. Sheiner LB, Beal SL. Some suggestions for measuring predictive performance. J Pharmacokinet Biopharm 1981;9(4):503-512.

9. US Preventive Services Task Force, editors. Guide to preventive services: report of the US Preventive Services Task Force. 2nd ed. Baltimore (MD): Williams \& Wilkins; 1996.

10. Tod M, Padoin C, Louchahi K, Moreau-Tod B, Petitjean O, Perret G. Application of optimal sampling theory to the determination of metacycline pharmacokinetic parameters: effect of model misspecification. I Pharmacokinet Biopharm 1994;22(2):129-146.

11. Suarez-Kurtz G, Ribeiro FM, Vicente FL, Struchiner CJ. Development and validation of limited-sampling strategies for predicting amoxicillin pharmacokinetic and pharmacodynamic parameters. Antimcrob Agents Chemother 2001;45(11):3029-3036.

12. Suarez-Kurtz G, Bozza FA, Vicente FL, Ponte CG, Stuchiner CJ. Limited-sampling strategy models for itraconazole and hydroxyitraconazole based on data from a bioequivalence study. Antimicrob Agents Chemother 1999;43(1):134-140. 
13. Uematsu T. Population pharmacokinetic analysis of new aminoglycosides, astromicin and isepamicin, and evaluation of Bayesian prediction method for approximation of individual clearance of drug. Int J Clin Pharmacol Ther Toxicol 1993;31(12):606-610.

14. Drusano GL, Forrest A, Snyder MJ, Reed MD, Blumer JL. An evaluation of optimal sampling strategy and adaptive study design. Clin Pharmacol Ther 1988;44(2):232-238.

15. Drusano GL, Forrest A, Plaisance KI, Wade JC. A prospective evaluation of optimal sampling theory in the determination of the steady-state pharmacokinetics of piperacillin in febrile neutroprnic cancer patients. Clin Pharmacol Ther 1989;45(6):635-641.

16. Rodvold KA, Rotschafer JC, Gilliland SS, Guay DR, Vance-Bryan K. Bayesian forecasting of serum vancomycin concentrations with nonsteady-state sampling strategies. Ther Drug Monit 1994;16(1):37-41.

17. Capparelli E, Hochwald C, Rasmussen M, Parham A, Bradley J, Moya F. Population pharmacokinetics of cefepime in the neonate. Antimicrob Agents Chemother 2005;49(7):2760-2766.

18. Soy D, Aweeka FT, Church JA, Cunningham CK, Palumbo P, Kosel BW, et al.; Pediatric AIDS Clinical Trial Group (PACTG) Study P1005 Investigators. Population pharmacokinetics of enfuvirtide in pediatric patients with human immunodeficiency virus: searching for exposure-response relationships. Clin Pharmacol Ther 2003;74(6):569-580.

19. Terziivanov D, Atanasova I, Dimitrova V. Population pharmacokinetics of ciprofloxacin in patients with liver impairments analyzed by NPEM2 algorithm—a retrospective study. Int J Clin Pharmacol Ther 1998;36(7):376-382

20. Kisor DF, Watling SM, Zarowitz BJ, Jelliffe RW. Population pharmacokinetics of gentamicin. Use of the nonparametric expectation maximisation (NPEM) algorithm. Clin Pharmacokinet 1992;23(1):62-68.

21. Noormohamed SE, Henry WK, Rhame FS, Balfour HH Jr, Fletcher CV. Strategies for control of zidovudine concentrations in serum. Antimicrob Agents Chemother 1995;39(12):2792-2797.

22. Yuen GJ, Drusano GL, Forrest A, Plaisance K, Caplan ES. Prospective use of optimal sampling theory: steady-state ciprofloxacin pharmacokinetics in critically ill trauma patients. Clin Pharmacol Ther 1989;46(4):451-457.

23. Amsden GW, Ballow $\mathrm{CH}$. Prospective validation of an optimal sparse plasma-sampling strategy for estimating ciprofloxacin pharmacokinetics. Pharmacotherapy 1996;16(5):937-941.

24. Mueller BU, Pizzo PA, Farley M, Husson RM, Goldsmith J, Kovacs A, et al. Pharmacokinetic evaluation of the combination of zidovudine and didanosine in children with human immunodeficiency virus infection. $J$ Pediatr 1994;125(1):142-146.

25. Burger DM, Meenhorst PL, ten Napel CHH, Mulder JW, Henrichs JH, Frissen PHJ, et al. Limited sampling models for the antiretroviral agent didanosine. Eur J Pharm Sci 1995;3(1):7-13.

26. Burger DM, Van Warmerdam LJC, Rosing H, et al. Development and validation of limited-sampling models for the antiretroviral agent zidovudine. Drug Invest 1993;6:189-197.

27. Veldkamp AI, van Heeswijk RP, Mulder JW, Meenhorst PL, Hoetelmans $\mathrm{RM}$, Lange JM, et al. Limited sampling strategies for the estimation of the systemic exposure to the HIV-1 nonnucleoside reverse transcriptase inhibitor nevirapine. Ther Drug Monit 2001;23(6):606-611.

28. Payen S, Serreau R, Munck A, Aujard Y, Aigrain Y, Bressolle F, et al. Population pharmacokinetics of ciprofloxacin in pediatric and adolescent patients with acute infections. Antimicrob Agents Chemother 2003:47(10):3170-3178.

29. López-Cortés LF, Ruiz-Valderas R, Marin-Niebla A, Pascual-Carrasco R, Rodriguez-Diez $\mathrm{M}$, et al. Therapeutic drug monitoring of efavirenz: trough levels cannot be estimated on the basis of earlier plasma determinations. J Acquir Immune Defic Syndr 2005;39(5):551-556.

30. Regazzi MB, Tinelli C, Villani P, Cusato M, De Silvestri A, Briganti E, et al. Limited sampling strategy for the estimation of systemic exposure to the protease inhibitor nelfinavir. Ther Drug Monit 2005;27(5):571-575.

31. Burstein AH, Gal P, Forrest A. Evaluation of a sparse sampling strategy for determining vancomycin pharmacokinetics in preterm neonates: application of optimal sampling theory. Ann Pharmacother 1997;31(9):980-983.

32. Balis FM, Pizzo PA, Butler KM, Hawkins ME, Brouwers P, Husson RN, et al. Clinical pharmacology of $2^{\prime}, 3^{\prime}$-dideoxyinosine in human immunodeficiency virus-infected children. J Infect Dis 1992;165(1):99-104.

33. Mueller BU, Lewis LL, Yuen GJ, Farley M, Keller A, Church JA, et al. Serum and cerebrospinal fluid pharmacokinetics of intravenous and oral lamivudine in human immunodeficiency virus-infected children. Antimicrob Agents Chemother 1998;42(12):3187-3192.
34. Alexander CS, Montaner JS, Asselin JJ, Ting L, McNabb K, Harris M, et al. Simplification of therapeutic drug monitoring for twice-daily regimens of lopinavir/ritonavir for HIV infection. Ther Drug Monit 2004;26(6):516-523

35. Vinks AATTM, Mouton JW, Touw DJ, Heijerman HG, Danhof M, Bakker W. Population pharmacokinetics of ceftazidime in cystic fibrosis patients analyzed by using a nonparametric algorithm and optimal sampling strategy. Antimicrob Agents Chemother 1996;40(5):1091-1097.

36. Dickinson L, Back D, Pozniak A, Khoo S, Boffito M. Limited-sampling strategy for the prediction of boosted hard-gel saquinavir exposure at a dosage of 1000/100 mg twice daily in human immunodeficiency virusinfected individuals. Ther Drug Monit 2007;29(3):361-367.

37. Preston SL, Drusano GL. Nonparametric expectation maximization population modeling of ganciclovir. J Clin Pharmacol 1996;36(4):301-310.

38. Kashuba AMD, Ballow CH, Forrest A. Development and evaluation of a Bayesian pharmacokinetic estimator and optimal, sparse sampling strategies for ceftazidime. Antimicrob Agents Chemother 1996;40(8):1860-1865.

39. Regazzi MB, Villani P, Maserati R, Seminari E, Pan A, LoCaputo F, et al. Clinical pharmacokinetics of nelfinavir combined with efavirenz and stavudine during rescue treatment of heavily pretreated HIV-infected patients. J Antimicrob Chemother 2000;45(3):343-347.

40. Perlman DC, Segal Y, Rosenkranz S, Rainey PM, Peloquin CA, Remmel $\mathrm{RP}$, et al. The clinical pharmacokinetics of pyrazinamide in HIV-infected persons with tuberculosis. Clin Infect Dis 2004;38(4):556-564.

41. Ariano RE, Zelenitsky SA, Nyhlén A, Sitar DS. An evaluation of an optimal sampling strategy for meropenem in febrile neutropenics. J Clin Pharmacol 2005;45(7):832-835.

42. Andrés I, López R, Pou L, Piñol F, Pascual C. Vancomycin monitoring: one or two serum levels? Ther Drug Monit 1997;19(6):614-619.

43. Forrest A, Ballow CH, Nix DE, Birmingham MC, Schentag JJ. Development of a population pharmacokinetic model and optimal sampling strategies for intravenous ciprofloxacin. Antimicrob Agents Chemother 1993;37(5):1065-1072.

44. Chatelut E, Rostaing L, Grégoire N, Payen JL, Pujol A, Izopet J, et al. A pharmacokinetic model for alpha interferon administered subcutaneously. Br J Clin Pharmacol 1999;47(4):365-371.

45. Dahri K, Ensom MHH. Efavirenz and nevirapine in HIV-1 infection: is there a role for clinical pharmacokinetic monitoring? Clin Pharmacokinet 2007;46(2):109-132.

46. Back D, Gibbons S, Khoo S. An update on therapeutic drug monitoring for antiretroviral drugs. Ther Drug Monit 2006;28(3):468-473.

47. Department of Health and Human Services, Panel on Antiretroviral Guidelines for Adult and Adolescents. Guidelines for the use of antiretroviral agents in HIV-1-infected adults and adolescents. Washington (DC): Department of Health and Human Services; 2008 [cited 2008 Mar 20]. Available from: http://www.aidsinfo.nih.gov/ContentFiles/ AdultandAdolescentGL.pdf

48. Rybak MJ. The pharmacokinetic and pharmacodynamic properties of vancomycin. Clin Infect Dis 2006;42 Suppl 1:S35-S39.

Denise A Sprague, BSc(Pharm), ACPR, PharmD, is a Clinical Pharmacy Specialist-Infectious Diseases, Kelowna General Hospital, Kelowna, British Columbia.

Mary H H Ensom, PharmD, FASHP, FCCP, FCSHP, FCAHS, is Professor and Director, Doctor of Pharmacy Program, Faculty of Pharmaceutical Sciences, and Distinguished University Scholar, The University of British Columbia, and Clinical Pharmacy Specialist with the Children's and Women's Health Centre of British Columbia, Vancouver, British Columbia. She is also the Editor of the CJHP.

\section{Address correspondence to:}

Dr Mary H H Ensom

Pharmacy Department (OB7)

Children's and Women's Health Centre of British Columbia

4500 Oak Street

Vancouver BC V6H 3N1

e-mail: ensom@interchange.ubc.ca 